\title{
Some Aspects of the Theory of Schrödinger Operators *
}

\author{
Barry Simon \\ Division of Physics, Mathematics and Astronomy \\ California Institute of Technology \\ Pasadena, California 91125
}

In these notes, we will survey a part of theory of the operator $-\Delta+V$. More extensive surveys can be found in $[1,2,3]$ and in $[4]$.

\section{Self-adjointness, properties of eigenfunctions and all that}

There is an enormous literature on the basic issue of giving a domain where $-\Delta+V$ is self-adjoint or essentially self-adjoint. To a large extent, I think one can single out two results as the most important: (1) The basic perturbation results of Kato-Rellich which accomodate virtually all cases of physical interest (2) "Kato's inequality," which, at least among positive $V$, is definitive. We will describe the first result briefly (for background on definition of selfadjoint, etc., see $[5,6,7]$; for a discussion of Kato's inequality, see $[1,8,9,10])$. Theorem 1.1 (The Kato-Rellich theorem $[11,12]$ ) Let $A$ be a self-adjoint operator on a Hilbert space, $\not$, and let $B$ be symmetric. Suppose that $D(B) \supset D(A)$ and for some $a<1$ and $b<\infty$,

$$
\left\|B_{\varphi}\right\| \leq a\|A \varphi\|+b\|\varphi\|
$$

for all $\varphi \in D(A)$. Then $A+B$ is self-adjoint on $D(A)$ and any core for $A$ is a core for $A+B$.

For a proof, see [1], pp. 162-163.

To apply this to $-\Delta+V$, we set $A=-\Delta, B=V$ and study (1.1). In this form, (1.1) is related to Sobolev estimates. Kato studied when (1.1) held in terms of $L^{P}$-spaces a point of view I long preferred, but I have come around to prefer a point of view introduced by Stummel [13].

Definition Fix $\nu \geq 4$, and $0<\alpha<4$ and 1et $S_{\alpha}^{(\nu)}$ be the set of functions, $V$, on $R^{\nu}$ obeying

* Research partially supported by USNSF grant MCS-81-20833 


$$
\sup _{x} \int_{|y-x| \leq 1}|x-y|^{-(\nu-4+\alpha)}|V(y)|^{2} d y<\infty
$$

If $\nu \leq 3$, we define $S_{\alpha}^{(\nu)}$ in terms of (1.2) with $|x-y|^{-(\nu-4+\alpha)}$ replaced by 1 (independently of $\alpha$ ).

With these definitions, it is not hard to prove the following pair of results (see Stumme1 [13]).

Theorem 1.2 If $V \in S_{\alpha}^{(\nu)}$, then (1.1) holds on $D(-\Delta)$ where $B=V, A=-\Delta$ and a can be taken arbitrarily close to zero.

Theorem 1.3 If $g \in S_{\alpha}^{(\mu)}, \nu>\mu$ and $V(x)=g(\pi x)$ where $\pi$ is a linear map of $R^{\nu}$ onto $R^{\mu}$, then $V \in S_{\alpha}^{(\nu)}$.

Thm. 1.2 is proven by noting that for $\nu \geq 4$, the integral of $\left(-\Delta+\kappa^{2}\right)^{-2}$ goes to $|x-y|^{-(\nu-4)}$ for $|x-y|$ smal1 and as $e^{-k|x-y|}$ for $|x-y|$ large and for $\nu \leq 3$, the kernel is bounded at sma11 distances. As a result, $\left\|V\left(-\Delta+\kappa^{2}\right)^{-2} V\right\| \rightarrow 0$ as $k \rightarrow \infty$. Theorem 1.3 follows by noting that $|x-y|^{-(\nu-4+\alpha)}$ integrated over $\nu-\mu$ variables (and cutoff at large distances) is bounded by $|x-y|-(\mu-4+\alpha)$.

The most important special case of Thm. 1.3 is to take $\mu$ fixed $(\mu=3$ is the physical case), $\nu=\mu N$, write a point in $R^{\nu}$ as $x=\left(x_{1}, \ldots, x_{N}\right)$ with $x_{j} \in R^{\mu}$ and let $T x=x_{i}-x_{j}$. Thus picking, for all pairs $i, j$, a function $u_{i j} \in S_{\alpha}^{(\mu)}$ and letting $v_{i j}(x)=v_{i j}\left(x_{i}-x_{j}\right)$, we see that $v_{i j} \in s_{\alpha}^{(\nu)}$. Therefore, the operator

$$
\tilde{H}=\tilde{H}_{0}+V ; \tilde{H}_{0}=\sum_{j=1}^{N}-\left(2 m_{j}\right)^{-1} \Delta_{j} ; V=\sum_{i<j} V_{i j} .
$$

called an $\mathrm{N}$-body Homiltonian obeys

Theorem 1.4 Any N-body Hamiltonian with $v_{i j} \in S_{\alpha}^{(\mu)}$ defines an operator $\tilde{H}$ self-adjoint on $D(-\Delta)$ and essentially self-adjoint on $C_{0}^{\infty}\left(R^{\nu}\right)$.

We used $\tilde{H}$ for the operator in (1.3) because there is a closely related operator, $H$, on $L^{2}\left(R^{\mu(N-1)}\right)$ called the operator with center of mass removed. Here are two ways of understanding this change: 
(1) Let $R=\sum m_{i} x_{i} / \sum m_{i}$ and 1et $\varsigma_{1}, \ldots, \varsigma_{N-1}$ be $N-1$ additiona 1 $\mu$-component coordinates (i.e. linear functions of the $x^{\prime} s$ ), so that (i) $\zeta_{j}$ is invariant under $x_{j} \rightarrow x_{j}+a$ for any a (ii) $x_{j} \rightarrow R, \zeta_{j}$ is an invertible transformation. For example, one might take

$$
S_{j}=r_{j}-r_{N} \quad j=1, \ldots, N-1
$$

Then by writing $R^{\mu N}=R^{\mu} \times R^{\mu(N-1)}$ by the coordinates $R, \zeta, L^{2}\left(R^{\mu N}\right)$ decomposes into $f_{\mathrm{cm}} \otimes f \equiv L^{2}\left(R^{\mu}\right) \otimes L^{2}\left(R^{\mu}(N-1)\right.$ ) (functions of $R$ tensored by functions of $\zeta$ ). Under this decomposition

$$
\tilde{H}=H_{0, c m} \otimes 1+1 \otimes H
$$

where $H_{0, c m}=-2\left(\sum m_{i}\right)^{-1} \Delta_{R}$ and $H=H_{0}+V$. The precise form of $H_{0}$ depends on the choice of local coordinates. For example, in the coordinate system (1.4),

$$
H_{0}=-\sum_{j=1}^{N-1}\left(2 \mu_{j}\right)^{-1} \Delta_{\zeta_{j}}+m_{N}^{-1} \sum_{i<j} \nabla_{i} \cdot \nabla_{j}
$$

with $\mu_{j}=\left(m_{N}^{-1}+m_{j}^{-1}\right)^{-1}$.

(2) $([14,15])$ View $\tilde{H}_{0}$ as one half the Laplace Beltrami operator associated to the metric $\|d x\|^{2}=\sum m_{j}\left(d x_{j}\right)^{2}$. Let $x=\left\{x_{i} \sum \sum m_{j} x_{i}=0\right\}$. Then in the metric, $x^{\perp}=\left\{x \mid x_{1}=x_{2}=\cdots=x_{N}\right\}, z_{c m}=L^{2}\left(X^{\perp}\right), y=L^{2}(X)$ and $H_{0}$ is just the LaplaceBeltrami operator on $X$ in the induced metric.

For later purposes, we introduce some additional notation to describe $\mathrm{N}$-body systems. A partition of $\{1, \ldots, N\}$, i.e. a family $C_{7}, \ldots, C_{k}$ of disjoint subsets of $\{1, \ldots, N\}$ which exhaust $\{1, \ldots, N\}$ is called a cluster decomosition. We write $a=\left\{C_{1}, \ldots, C_{k}\right\} ; k \equiv \#(a)$. The family of cluster decompositions is important because in various aspects of the study of $\mathrm{N}$-body Hamiltonians, one expects that we want to analyze what happens as $\|x\| \rightarrow \infty$ with $\sum m_{i} x_{i}=0$. This happens if the system breaks up into distinct clusters; i.e. we can find numbers $R_{7}, \ldots, R_{k}$ and a decomposition a so $\left|x_{i}-R_{j}\right|$ stays bounded if $i \in C_{j}$ and so each $\left|R_{i}-R_{j}\right|$ goes to infinity.

Given a, we pick coordinates $\varsigma_{1}, \ldots, \varsigma_{k}$ involving differences of center of 
mass of clusters in a, and "internal coordinates," $\varsigma^{1}, \ldots, \zeta^{\mathrm{N}-1-\mathrm{k}}$, i.e. coordinates left invariant by the transformations $x_{i} \rightarrow x_{i}+R_{j(i)}$ where $j(i)$ is that $j$ with $x_{i} \in C_{j}$. (Put different1y, $\zeta^{1}, \ldots, \zeta^{N-1-k}$ are coordinates for the plane $x^{a}=\left\{x \mid \sum_{i \in C_{j}} m_{i} x_{i}=0, a 11 j\right\}$ and $\zeta_{1}, \ldots, \zeta_{k}$ for $i t s$ orthogonal complement, $x_{a}$, in $\left.x\right)$. If we decompose $z=z^{a} \otimes z_{a}$ corresponding to functions of $\zeta^{a}$ and $\zeta_{a}$ (i.e. $y^{a}=L^{2}\left(x^{a}\right), w_{a}=L^{2}\left(X_{a}\right)$ ), then we have

$$
V=V(a)+I(a) ; I(a)=\sum_{(i j) \not \subset a} V_{i j} ; V(a)=\sum_{(i j) \subset a} V_{i j}
$$

(where $(i j) \subset$ a means $i$ and $j$ are in the same cluster), and

$$
\begin{gathered}
H \equiv H(a)+I(a) \\
H(a)=H^{a} \otimes I+I \otimes T_{a}
\end{gathered}
$$

where $T_{a}$ has no potentials and is exactly the kinetic energy of relative motion of the clusters. Eigenvalues of $H^{a}$ with $\#(a) \geq 2$ are called thresholds.

[16] contains extensive discussion of properties of eigenfunctions of $-\Delta+V$. Here we state some of the most important results. For many purposes, the natural class of potentials, $V$, for this study is $K^{\nu}$ defined by:

Definition Let $\nu \geq 3 ; \quad \forall \in K^{\nu}$ if and only if

$$
\lim _{\alpha \rightarrow 0} \sup _{x} \int_{|x-y| \leq a}|x-y|^{-(\nu-2)}|v(y)| d^{\nu} y=0
$$

If $\nu=2, K^{\nu}$ is defined with $|x-y|^{-(\nu-2)}$ replaced by $\ln \left(|x-y|^{-1}\right)$ and if $\nu=1, V \in K^{\nu}$ if and only if $\sup _{x} \int_{|x-y| \leq 1}|V(y)| d^{\nu} y<\infty$.

$V \in K^{\nu}$ does not imply that $-\Delta+V$ is essentially self-adjoint on $C_{0}^{\infty}$, but one can [16] always define a self-adjoint operator " $-\Delta^{+} V^{\prime}$ by a method of forms: This meaning agrees with that obtained by closing the operator on $C_{0}^{\infty}$ in case the operator sum is self-adjoint there. The following three results are discussed 
(either proofs or references given) in [16]. If $H u=E u$, then $\left(-\Delta^{+}(V-E)\right) u=0$, so by changing $V$, we can look at functions with $\mathrm{Hu}=\mathrm{O}$ and obtain information on general eigenfunctions.

Theorem 1.5 (Sobolev estimates for $H$ ) Let $H=-\Delta+V$ with $V_{-} \equiv \max (-V, 0)$ in $k^{\nu}$ and $V_{+}=\max (V, 0)$ in $K_{10 c}^{\nu}$. Let $k>\nu / 4$. Then any function in $D\left(\left[\left.H\right|^{k}\right)\right.$ is a bounded continuous function.

Theorem 1.6 (Subsolution estimate for $H$ ) Let $H$ obey the hypotheses of Thm. 1.5. Let $u$ obey $\mathrm{Hu}=0$ in distributional sense ( $u$ not necessarily in $L^{2}$ ). Then

$$
|u(x)| \leq C \int_{|x-y| \leq 1}|u(y)| d^{2} y
$$

for a constant $C$ depending only on $K^{\nu}$ norms of $V_{-}$.

Theorem 1.7 (Harnack's inequality for $H$ ) Let $V \in K_{10 c}^{\nu}$. Let $\Omega$ be a bounded open set and $K$ compact in $\Omega$. Then, there is a constant $C$ depending only on $k^{\nu}$ norms of $V x_{\Omega}$ so that every solution, $u$, of $H u=0$ in $\Omega$ with $u$ nonnegative on $\Omega$, obeys

$$
C^{-1} u(x) \leq u(y) \leq C u(x)
$$

for al1 $x, y$.

We will not indicate in detail the proofs of the last two theorems. In many ways, the key is the study of the Poisson kernel for $H$, i.e. for a small open bal1, $B$, about a point $x$, one can study the map, $M_{V}^{B}$ from continuous functions $f$ on $\partial B$ to functions on $B$ defined by $M_{V}^{B}(f)=u$ obeys $H u=0$ in distributional sense on $B$ and $u(x) \rightarrow f^{\prime}(y)$ as $x \rightarrow y$ on $\partial B$. It happens that $\left(M_{V}^{B} f\right)(x)=\int_{\partial B} P_{V}^{B}(x, y) f(y) d w(y)$. The last two theorems are proven by showing that $P$ is bounded above and away from zero as $x$ runs through a compact subset of $B$ and $y$ runs through $\partial B$. This is precisely what Aizenman-Simon [17] do. Recently, Zhao [18] and Brossard [19] have actually proven more subtle estimates showing that $P_{V}^{B}(x, y) / P_{V=0}^{B}(x, y)$ is bounded above and below uniformly in $x$ and $y$ (i.e. they show the boundary behavior of $P$ is essentially $V$ independent). 


\section{Bound state problems}

"Bound states" is the name given to eigenfunctions of eigenvalues in the discrete spectrum (isolated points of the spectrum of finite multiplicity). There are various aspects of the study of eigenfunctions: (i) Identify $\sigma_{\text {ess }}(H)$ $\left(=\sigma(H) \backslash \sigma_{\operatorname{disc}}(H)\right)$ (ii) Let $\mathrm{N}$ denote the sum of the dimensions of the eigenspaces associated to all points in $\sigma_{\text {disc }}$. Is $N$ finite or infinite? (This is the same as asking if $\#\left(\sigma_{\text {disc }}\right)$ is finite or infinite.) (iii) If $N$ is finite, can one obtain effective bounds on it? (iv) When is $N=0$ ?

For two body systems, $-\Delta+V$ with $V$ decaying at $\infty$, there is a large literature on these questions, summarized in [20]. We will single out two results for special mention, but first we need to find $\sigma_{\text {ess }}(-\Delta+V)$ in this case.

Definition Let $A$ be a self-adjoint operator. $B$ is called A-compact if and only if $D(B) \supset D(A)$ and $B(A+i)^{-1}$ is compact.

The methods of the proof of Thm.1.2 imply easily that

Proposition 2.1 If $\nu \leq 3$ and $\lim _{|x| \rightarrow \infty} \int_{|y-x| \leq 1}|v(y)|^{2} d y=0$ or if $\nu \geq 4$ and $\lim _{|x| \rightarrow \infty} \int_{|x-y| \leq \leq}|y-x|^{-(\nu-4+\alpha)}|V(y)|^{2} d y=0$ for some $\alpha>0$, then $V$ is $-\Delta$-compact. We write $S_{\text {comp }}^{\nu}$ for the $V^{\prime}$ 's given in Prop. 2.1.

Proposition 2.2 If $A$ is self-adjoint, and if B is A-compact and symmetric, then $\sigma_{\text {ess }}(A \div B)=\sigma_{\text {ess }}(A)$.

Proof A simple theorem of Weyl (see [3]) says that $E \in \sigma_{\text {ess }}(C)$ if and only if there exists a sequence of vectors $\varphi_{n} \in D(C)$ with $\varphi_{n} \rightarrow 0$ weakly and $\left\|(C-E)_{n}\right\| \rightarrow 0,\left\|\varphi_{n}\right\| \rightarrow 0$. Given $E \in \sigma_{e s s}(A)$, find such a sequence, let $\Psi_{n}=$ $\left(E^{2}+1\right)\left(A^{2}+1\right)^{-1} \varphi_{n}$. It is not hard to show that $\psi_{n} \rightarrow 0$ weak1y, $\left\|(A+B-E) \psi_{n}\right\| \rightarrow 0,\left\|\Psi_{n}\right\| \rightarrow 1$. Thus, $E \in \sigma_{\text {ess }}(A+B)$ and we conclude that $\sigma_{\text {ess }}(A) \subset \sigma_{\text {ess }}(A+B)$. Using $(A+B+i)^{-1}=$ $(A+i)^{-1}\left(1+B(A+i)^{-1}\right)^{-1}$, one can show that $B$ is $(A+B)$-compact. Thus, $\sigma_{\text {ess }}(A+B) \subset$ $\sigma_{\text {ess }}(A)$ by repeating the above argument.

Corollary 2.3 If $V$ lies in $S_{\text {comp }}^{\nu}$, then $\sigma_{\text {ess }}(-\Delta+V)=[0, \infty)$. 
We return now to $N$ for $-\Delta+V$ which we denote by $N(V)$. We want to single out two results:

Theorem 2.4 (Quasiclassical bounds on $N(V)$ ) Let $\nu \geq 3$. There is a universal constant $C_{\nu}$ so for al1 $V \in L^{\nu / 2}$,

$$
N(V) \leq C_{V} \int \| v(x) \int^{\nu / 2} d^{\nu} x
$$

This theorem is particularly important because the semiclassical approximation for $N(V)$ is to take the volume in phase space where $p^{2}+V(x)$ is negative and divide it by $(2 \pi)^{\nu}$ (for $\hbar=1$, so $h$ is $2 \pi$ ). Thus if $V(x) \leq 0$ :

$$
N_{c_{\ell}}(V)=\frac{\tau_{\nu}}{(2 \pi)^{\nu}} \int\left[\left.V(x)\right|^{\nu / 2} d^{\nu} x\right.
$$

where $\tau_{\nu}$ is the volume of the unit sphere in $R^{\nu}$. As a result, Thm. 2.3 says that the quantum $\mathrm{N}(V)$ is bounded by a multiple of $\mathrm{N}_{\mathrm{Cl}}(V)$. There is also a connection with Sobolev estimates (see $[21,22]$ ). Thm. 2.1 was proven independently (with different $C_{\nu}$ ) by Rosenbljum [23], Cwicke1 [24] and Lieb [25] (see [21,26] for expositions of $[25,24]$ ) with newer proofs by Li-Yau [27] and FeffermanPhong [28]. Theorem 2.4 is in some sense especially accurate for "large" $V$ : Theorem 2.5 (Quasiclassical 1imit for $N(V)$ ). Let $\nu \geq 3, V \in L^{\nu / 2}$. Then

$$
\lim _{\lambda \rightarrow \infty} N(\lambda V) / N_{C 2}(\lambda V)=1
$$

Since $-\Delta+\lambda V=\left(-\lambda^{-1} \Delta+V\right) \lambda$, the $\lambda \rightarrow \infty$ limit is. "equivalent" to the $\hbar \rightarrow 0$ limit, which is "why" the semiclassical result is asymptotically correct. Thm. 2.4 is used to show that Thm. 2.5 need only be proven when $V \in C_{0}^{\infty}$ where Thm. 2.5 was proven independently by Birman-Borzov [29], Martin [30] and Tamura [31] (see [3,21] for pedagogic discussions). A multiparticle analog of Thm. 2.4 can be found in [32].

Now we want to describe some results on bound states for multiparticle systems. The first basic result describes $\sigma_{\text {ess }}(H)$. We first use the partition 
notation described in Section 1.

Theorem 2.6 (HVZ theorem) Let $H$ be the Hamiltonian (with C.M. motion removed) of an $\mathrm{N}$-body system on $\mathrm{L}^{2}\left(\mathrm{R}^{\mu(\mathrm{N}-\mathrm{T})}\right)$ with two body potential in $\mathrm{S}_{\text {comp }}^{\mu}$. Let

$$
\Sigma=\inf _{a \| \#(a) \geq 2}[\min \sigma(H(a))]
$$

Then $\sigma_{\text {ess }}(H)=[\Sigma, \infty)$.

In order to understand this result, it is useful to know

Theorem 2.7 (Persson's theorem [33]) Let $V_{-} \in K^{\nu}, V_{+} \approx K_{70 c}^{\nu}$. Then

$$
\inf \sigma_{e s s}(-\Delta+V)=\lim _{R \rightarrow \infty} \inf \left\{\left(\varphi,(-\Delta+V)_{\varphi}\right)\left\|\varphi \in C_{0}^{\infty}\left(R^{\nu}\right) ;\right\| \varphi \|=1 ; \operatorname{supp} \varphi \subset\{x\|\| x \mid>R\}\right\}
$$

For a proof, see also Agmon $[34,35]$ or Cycon et a1. [4]. What Persson's theorem suggests is that essential spectrum is associated with vectors living near infinity (this is basically because $(\mathrm{H}+\mathrm{i})^{-1}$ times the characteristic function of a bounded set is compact). Thus, in the $\mathrm{N}$-body case, essential spectrum is associated with states near infinity where the system must break up into two or more subsets. Thus, one should expect

$$
\sigma_{\text {ess }}(H)=\underset{a ! H(a) \geq 2}{U} \sigma(H(a))
$$

which is just a restatement of Thm. 2.6.

Thm. 2.6 has two parts in a natural sense: (i) $[\Sigma, \infty) \subset \sigma(H)$ and (ii) $\sigma(H) \cap(-\infty, \Sigma)$ is discrete. (i) is the "easy" half and (ii) will be what we concentrate on (see e.g. Garding [36] for the "easy" ha7f). The name HVZ recognizes contributions of Hunziker [37], van Winter [38] and Zhislin [39]. Zhislin used geometric ideas together with rather extensive machinery, so for some years the integral equation proof of van Winter and Zhislin was considered the more elementary (see e.g. [3] for that proof), but with the work of Enss [40] and Simon [41], the geometric proof has come into fashion, and it is Sigal's version of it [42] that we will sketch.

We begin with a basic result on localization called the "IMS localization formula" due to contributions of Ismigilov, Morgan, Simon and I.M. Sigal, who 
first appreciated its great usefulness.

Proposition 2.8 Let $\left\{j_{a}\right\}$ be a finite family of functions with distributional gradients in $L^{\infty}$ obeying $\sum j_{a}^{2}=1$. Let $H=-\Delta+V$ on $L^{2}\left(R^{\nu}\right)$ have $C_{0}^{\infty}$ as a form core. Then

$$
H=\Sigma j_{a} H j_{a}-\Sigma\left(\nabla j_{a}\right)^{2}
$$

Remark (2.1) is intended in the sense of expectation values with $\left(\varphi, j_{a} H j_{a} \varphi\right) \equiv$ $\left(j_{a} \varphi, H j_{a} \varphi\right)$. If the $j^{\prime} s$ are sufficiently smooth, it holds in operator sense.

Proof By a limiting argument, we can suppose the $j$ 's are $c^{\infty}$. Then $\left[j_{a},\left[j_{a}, H\right]\right]=\left[j_{a},\left[j_{a},-\Delta\right]\right]=-2\left(\nabla j_{a}\right)^{2}$. Thus

$$
\sum_{a} j_{a}^{2} H+H j_{a}^{2}=2 \underset{a}{\sum} j_{a} H j_{a}-2 \sum_{a} \nabla j_{a}^{2}
$$

which yields (2.1) given $\sum j_{a}^{2}=1$.

Next, we need the existence of a special partition of unity for $\mathrm{N}$-body system: A Ruelte-Simon partition of unity of an $\mathrm{N}$-body system is a set of functions $\left\{j_{a}\right\}$ on $X$ (the $C M=0$ space) labeled by partitions, $a$, with $\#(a)=2$ obeying (i) $j_{a}$ is $c^{\infty}$ (ii) $\sum j_{a}^{2}=1$ (iii) if $\lambda>1$ and $\|x\|>1$, then $j_{a}(\lambda x)=j_{a}(x)$ (iv) for some $c>0$,

$$
\left[\operatorname{supp} j_{a} \cap\{x\|\| x \|>1\}\right] \subset \underset{(i j) \not \subset a}{U}\left\{x_{1}\left\|x_{i}-x_{j}\right\| \geq C\|x\|\right\}
$$

Thus $j_{a}$ lives in the region where particles in different clusters of a are far from each other as $\|x\| \rightarrow \infty$. (The norm of $x$ is measured in any convenient way.) Proposition 2.9 Ruelle-Simon partitions of unity exist.

Sketch of proof Let $S_{a}=\left\{x\|\| x \|=1, x_{i}=x_{j}\right.$ for some $\left.(i j) \subset a\right\}$. We claim that $\bigcap_{a} S_{a}=\phi$. For if $\|x\|=1, x_{i} \neq x_{j}$ for some pair $i, j \quad\left(\right.$ since $\left.\sum m_{j} x_{i}=0\right)$. Let $a=\left\{C_{1}, C_{2}\right\}$ with $C_{1}=\left\{x \mid x=x_{i}\right\}$ and $C_{2}=\{1, \ldots, N\} \backslash C_{1}$. Then $x \notin S_{a}$. Since the $S_{a}$ are closed and $\bigcap_{a} S_{a}=\phi$, it is not hard to find $c^{\infty} \tilde{j}_{a}$ on $\{x\|\| x \|=1\}$ so that $\sum \tilde{j}_{a}^{2}=1$ and $\tilde{j}_{a}$ vanishes in a neighborhood of $S_{a}$. Now let $j_{a}(x)=$ $\tilde{j}_{a}(x /\|x\|)$ if $\|x\| \geq 1$ and continued to be smooth near 0 . 
One can actually estimate the constant C; see [41].

Proposition 2.10 Let $H$ be an N-body Hamiltonian of the type described in Thm. 2.6, and let $\left\{j_{a}\right\}$ be a Ruelle-Simon partition of unity. Then:

(a) $\left(\nabla j_{a}\right)^{2}$ is $H$-compact for any a

(b) $I(a) j_{a}$ is $H$-compact for any a.

Proof $\left(\nabla j_{a}\right)^{2}$ is bounded and goes to zero at $\infty$ (as $\left\{\times \ell^{-2}\right.$ ) so (a) is easy. If $(i j) \not \subset a,\left|x_{i}-x_{j}\right| \rightarrow \infty$ as $|x| \rightarrow \infty$, so it is not hard to show that $v_{i j} j_{a}$ is H-compact (see e.g. [41]).

We are now ready for

Proof of Thm. 2.6 [42] (Hard direction) Pick a Ruelle-Simon partition of unity. Write

$$
\begin{aligned}
& H=\hat{H}+W, \hat{H}=\sum_{\#(a)=2} j_{a} H(a) j_{a} \\
& W=\sum j_{a} I(a) j_{a}-\sum\left(\nabla j_{a}\right)^{2}
\end{aligned}
$$

$W$ is $H$-compact, so $\sigma_{\text {ess }}(H)=\sigma_{\text {ess }}(\hat{H})$ by Prop. 2.2. Since $H(a) \geq \sum$ for all a, we have for any $\varphi$ in $L^{2}$ that

$$
\left(\varphi, \hat{H}_{\varphi}\right) \geq \sum_{a}\left(j_{a}^{2} \varphi, \sum \varphi\right)=\sum(\varphi, \varphi)
$$

so $\sigma_{\text {ess }}(\hat{H}) \subset \sigma(H) \subset[\Sigma, \infty)$.

These geometric methods have been extremely useful in studying bound state problems in $\mathrm{N}$-body systems. Here are some results which we quote without detailed proofs:

Theorem 2.11 Let $\nu \geq 3$. Let $H$ be the Hamiltonian of an $N$-body system with potentials $V_{i j}$ obeying $\left|V_{i j}(x)\right| \leq c(1+|x|)^{-2-\varepsilon}$. Suppose that the bottom of the continuum is two body in the sense that

$$
\inf _{\#(a) \geq 3} \sigma(H(a))>\inf _{\#(a) \geq 2} \sigma(H(a))
$$


Then $H$ has only a finite discrete spectrum.

Theorems of this genre go back to Zhislin and collaborators, see e.g. $[43,44]$. This result is proven by geometric means in Sigal [42].

Theorem 2.12 For any $N, Z$ let $H(N, Z)$ be the operator on $L_{a}^{2}\left(R^{3 N}\right.$ ) ( function on $R^{3 N}=\left\{x=\left(x_{1}, \ldots, x_{N}\right)\left\{x_{i} \in R^{3}\right\}\right.$ antisymmetric in the $x_{i}$ 's) given by

$$
H(N, Z)=\sum_{i=1}^{N}-\Delta_{i}-\frac{Z}{\left|x_{i}\right|}+\sum_{i \neq j} \frac{1}{\left|x_{i}-x_{j}\right|}
$$

Let $E(N, Z) \equiv$ inf $\operatorname{spec} H(N, Z)$. Then, there exists $N(Z)$, so that

$$
E(N+1, Z)=E(N, Z) \quad \text { if } N \geq N(Z)
$$

This result says that a nucleus of charge $Z$ bonds at most $N(Z)$ electrons (we will take $N(Z)$ to be the smallest $N(Z)$ obeying the above). Thm. 2.12 with $L_{a}^{2}$ replaced by $\mathrm{L}^{2}$ was proven by Ruskai [45]; Thm. 2.12 was proven by Sigal [42]. Recently, Lieb [46] has found an elegant direct proof that $N(Z) \leq 2 Z$ for a11 $Z$. Using Sigal's method, Lieb et al. [47] have proven that $\lim _{Z \rightarrow \infty} N(Z) / Z=1$. 


\section{The basic notions of scattering theory}

We will introduce here some of the simplest notions in scattering theory; Enss, in his lectures, will discuss much more involved ideas. See [2] for an extensive discussion of scattering theory.

Given $A, B$, we want to find pairs $\varphi, \psi$ so

$$
e^{-i t A_{\varphi}}-e^{-i t B_{\psi}} \rightarrow 0 \text { as } t \rightarrow+\infty
$$

It turns out that for general $B$, one shouldn't normally expect that a $\varphi$ exists for every $\psi$. For example, if $B \psi=0$, one must have $\varphi=\psi$ and $A \dot{\psi}=0$ for the limit (3.1) to occur. Thus, we only try to find $\varphi$ for $\psi \in H_{a} . c$. (B), the absolutely continuous subspace for B. Note (3.1) is equivalent to

$$
\varphi=\lim _{t \rightarrow \infty} e^{i t A^{-i t B}} \psi
$$

This motivates

Definition Given two self-adjoint operators, $A, B$ we say that the wave operators $\Omega^{ \pm}(A, B)$ exist if and only if $\operatorname{s}_{t \rightarrow \mp \infty} e^{i t A} e^{-i t B} P_{a . c .}(B)$ exists.

Notice that if $s$ is fixed and we replace $t$ by $t-s$, the limit is the same. Thus:

$$
e^{-i s A_{\Omega}^{ \pm}}(A, B)=\Omega^{ \pm}(A, B) e^{-i s B}
$$

This implies that $B$ restricted to $\operatorname{Ran} P_{a . c .}(B)$ and $A$ restricted to $\operatorname{Ran} \Omega^{ \pm}(A, B)$ are unitarily equivalent. In particular, $\operatorname{Ran} \Omega^{ \pm}(A, B) \subset \operatorname{Ran} P_{a}$.c. (A). It is clearly natural to single out:

Definition Let $\Omega^{ \pm}(A, B)$ exist. We say they are complete if and only if $\operatorname{Ran} \Omega^{ \pm}(A, B)=\operatorname{Ran} P_{a . c .}(A)$.

If $\Omega^{ \pm}$exist and are complete, then the association (3.1) sets up a one-one correspondence between $z_{a} . c .(A)$ and $H_{a} . c$. (B). Given the fact that (3.1) is symmetric in $A$ and $B$, it is not hard to show:

Proposition 4.1 Let $\Omega^{ \pm}(A, B)$ exist. Then, they are complete if and only if 
$\Omega^{ \pm}(B, A)$ exist.

Remark Deift-Simon [48] have an N-body analog of Prop. 4.1.

Proposition 4.1 suggests that one look for a condition symmetric in $A, B$ which implies that $\Omega^{ \pm}(A, B)$ exist. The strongest such result seems to be:

Theorem 4.2 Let $A, B$ be self-adjoint operators with $(A+i)^{-1}-(B+i)^{-1}$ compact and so that for any interval, $\Delta: E_{\Delta}(A)(A-B) E_{\Delta}(B)$ is trace class (where $E_{\Delta}(\cdot)$ is a spectral projection). Then $\Omega^{ \pm}(A, B)$ exist and are trace class.

Theorems of this genre go back to Kato and Rosenbljum, with later contributions of note by Kato, Birman, Pearson and Davies. This result follows from an observation of Davies [49] and a theorem of Birman which appears as Cor. 6.7 in [26].

As far as the abstract theory is concerned, Thm. 4.2 is quite elegant. However, in the concrete situation of $A=-\Delta+V$ and $\Omega=-\Delta$ on $L^{2}\left(R^{\nu}\right)$, it breaks down when $V$ decays more slowly than $|\times|^{-\nu}$ while one expects that $\Omega^{ \pm}(A, B)$ and are complete so long as $V$ only has $|x|^{-1-\varepsilon}$ decay. Various methods exist for proving that this compactness result (existence is easy, see [2], Sect. XI.4 and references therein):

(a) The method of weighted $L^{2}$ estimates developed by Agmon and Kuroda and discussed in Section XIII.8 of [3].

(b) The Enss method dišcussed in Section XI.17 of [2], and the book of Perry [50].

(c) Combining the Mourre estimates, to be described in the next section, with the smoothness techniques of Kato and Lavine (see e.g. Perry, Sigal, Simon [51]).

For $\mathrm{N}$-body systems, the notion of completeness requires a more elaborate definition. Let $\mathrm{H}^{\mathrm{a}}$ be the Hamiltonian describing internal motion for the clustering $\mathrm{a}$ and let $\mathrm{p}^{\mathrm{a}}$ denote the projection onto the span of the eigenvectors of $H^{a}$, and let $P(a)=P^{a} \otimes I$. One defines for any $a$

$$
\Omega_{a}^{ \pm}=\lim _{t \rightarrow \mp \infty} e^{i t H_{1}} e^{-i t H(a)} P(a)
$$

Under suitable hypotheses, it is not hard to show that $\Omega_{a}^{ \pm}$exist (see Thm. XI.35 in [2], but note the arguments there require modification if $\nu=1,2) . \Omega_{a}^{ \pm} \varphi=\eta$ is 
a state with $e^{-i t H_{\eta}}$ asymptotic as $t \rightarrow-\infty$ to a state with bound clusters in a moving relatively freely. It is thus reasonable and not hard to prove ([2], Thm. XI.36(b)) that

$$
\operatorname{Ran} \Omega_{\mathrm{a}}^{+} \perp \operatorname{Ran} \Omega_{\mathrm{b}}^{+} \quad \mathrm{a} \neq \mathrm{b}
$$

Completeness now reads

$$
L^{2}\left(R^{(N-1) \nu}\right)=\underset{a}{\oplus\left(\operatorname{Ran} \Omega_{a}^{ \pm}\right)}
$$

Notice that if $a_{1}$ is the unique clustering with $\#\left(a_{1}\right)=1 \quad\left(\right.$ so $H\left(a_{1}\right)=H$ ), then $\frac{ \pm}{a_{1}}=P\left(a_{1}\right)$ is the projection onto the point spectral subspace $H$, and that by the intertwining relation

$$
e^{i t H_{\Omega_{a}^{ \pm}}^{ \pm}}=\Omega_{a}^{ \pm} e^{i t H(a)}
$$

Ran $\Omega_{a}^{ \pm} \subset \xi_{a . c .}(H)$ if $\#(a) \geq 2$. Thus (3.4) implies that $H$ has empty singular continuous spectrum.

Thus far, there are fairly general results on three body completeness $[52,53,54]$ but only very specialized results for $\mathrm{N}$-body, see e.g. $[55,56,57,58]$. It has been emphasized to me by I.M. Sigal that the following result which extends an idea of Deift-Simon [48] should be very useful in a possible inductive proof of completeness:

Proposition 4.3 Let $\{A(a)\}_{\#}(a) \geq 2$ be bounded operators with $\sum_{a} A(a)=1$. Suppose that (i) Each $H^{a}$ with $H(a) \geq 2$ is complete (ii) The operators $\lim _{t \rightarrow F \infty} e^{i t H(a)} A(a) e^{-i t H_{P}}$ a.c. $(H) \equiv w_{a}^{ \pm}$ exist. (iii) $\ddot{H}_{\text {sing }}(H)=\phi$. Then $H$ is complete.

Proof Let $\eta \in \operatorname{Ran} P_{a . c .}(H)$. Then

$$
e^{-i t H_{\eta}}=\sum_{a} A(a) e^{-i t H_{\eta}} \sim \sum_{a} e^{-i t H(a)} W_{a}^{ \pm} \eta
$$

where $\sim$ means the difference goes to zero as $t \rightarrow \mp \infty$. Since $H^{a}$ is complete, $e^{-i t H(a)} \varphi$ is asymptotically a sum of vectors of the form $e^{-i t H(b)} P(b) \varphi_{b}$ with $b \leq a$, so we have completeness for $H$. 


\section{Mourre estimates}

Eric Mourre, in a deep paper [59], singled out certain estimates which he showed have important spectral consequences, and which he proved for a large class of two and three body systems. Perry, Sigal, Simon [51] then gave an involved proof of these estimates for general $\mathrm{N}$-body systems. Subsequently, Froese-Herbst [80] found a rather simple proof of these PSS results.

Let $H$ be a self-adjoint operator, and $A$ a second self-adjoint operator. We will not be explicit about all domains referring the reader to $[59,51]$ for explicit hypotheses. Under such hypotheses, one can define $-i[A, H]=B$ originally on a suitable core for $H$ and then as an "operator" from $D(H)$ to $D^{-1}(H)$ (equal abstract dual of $D(H))$ i.e. $(H+i)^{-1} B(H+i)^{-1}$ is a bounded operator. We say that $H$ obeys a Mourre estimate at a point $E_{0}$, if there is an open interval $\Delta$ about $E_{0}$ so that

$$
E_{\Delta} B E_{\Delta} \geq \alpha E_{\Delta}^{2}+E_{\Delta} K E_{\Delta}
$$

for a compoct operator $K$, and some $\alpha>0$.

Theorem 4.1 Under suitable domain hypotheses (including a bound on $[A, B]$ ), if a Mourre estimate holds for any $E_{0}$ I, an open interval, then

(i) $H$ has no singular continuous spectrum in $I$

(ii) In any compact $\mathrm{J} \subset \mathrm{I}$, there are finitely many eigenvalues of $\mathrm{H}$ and each eigenvalue has finite multiplicity.

(iii) For any compact $J \subset I$ and $\delta>0, \sup _{\|}\left\|(|A|+1)^{-\frac{1}{2}-\delta}(H-E-i \varepsilon)^{-1}(|A|+1)^{-\frac{1}{2}-\delta}\right\|$ $\underset{E \in J}{0 \leqslant<<1}$

$<\infty$.

The result is essentially due to Mourre [59], although the above include refinements of [51]. While we will not give the proof in detail, we note the basic idea behind ( $i i)$. Using the unstated domain conditions, one verifies a Virial theorem: If $H_{\varphi}=E_{\varphi}$, then $\left(\varphi, B_{\varphi}\right)=0$. Thus, if $H_{\varphi_{n}}=E_{n} \varphi_{n}$ with $E_{n} \rightarrow E_{0} \in \Delta$ and $\varphi_{n}$ is orthonormal, then, by (4.1)

$$
0 \geq \alpha\left\|\varphi_{n}\right\|^{2}+\left(\varphi_{n}, K \varphi_{n}\right)
$$


which is impossible since $K$ is compact and $\varphi_{n} \rightarrow 0$ weakly.

Mourre [61] (see also [62]) has also proven interesting propagation estimates when Mourre estimates hold.

When does an estimate like (4.1) hold? Mourre had the idea of taking $A=\frac{1}{2}(x \cdot p+p \cdot x)$ (with $\left.p=-i_{\nabla}\right)$, the generator of dilations. For two body operators, $H=H_{0}+V$

$$
-i[A, H]=2 H_{0}-x \cdot \nabla V=2 H+W
$$

where

$$
W=-x \cdot \nabla V-2 V
$$

If $K=E_{\Delta}(H) W E_{\Delta}(H)$ is compact, and if $\Delta=[a, b]$ with $a>0$, then $E_{\Delta} B E_{\Delta} \geq$ $2 a E_{\Delta}^{2}+E_{\Delta} K E_{\Delta}$, so a Mourre estimate holds.

Proposition 4.2 If $V=V_{1}+V_{2}$ with $V_{1}\left(H_{0}+i\right)^{-1}, x \cdot \nabla V_{1}\left(H_{0}+i\right)^{-1}$ and $\left(1+[x \mid) V_{2}\left(H_{0}+i\right)^{-1}\right.$ all compact, then a Mourre estimate holds for $A=\frac{1}{2}(x \cdot p+p \cdot x)$; $H=-\Delta+V$ and $E_{0}>0$.

Proof Note first that $D(H)=D\left(H_{0}\right)$, so $\left(H_{0}+i\right) E_{\Delta}(H)$ is bounded. Thus, $E_{\Delta} W_{1} E_{\Delta}$ is obviously compact as is $E_{\Delta} V_{2} E_{\Delta}$. As for $-i\left[A, V_{2}\right]$, we can write that as $-\sum_{i}\left[\nabla_{i}, x_{i} V_{2}\right]+\nu V_{2}$ and $E_{\Delta}\left[\left(\nabla_{i}\right)\left(x_{i} V_{2}\right)\right] E_{\Delta} \quad$ is compact.

Mourre [59] showed how to do this for three-body systems and then PSS [51] proved:

Theorem 4.3 If each $v_{i j}=v_{i j}^{(1)}+v_{i j}^{(2)}$, where as operators on $L^{2}\left(R^{\nu}\right), v^{(1)}\left(h_{0}+i\right)^{-1}$, $x \cdot \nabla V^{(1)}\left(h_{0}+i\right)^{-1}$ and $\left(1+\left[x[) V^{(2)}\left(h_{0}+i\right)^{-1}\right.\right.$ are all compact on $\left(h_{0}=-\Delta\right.$ on $\left.R^{\nu}\right)$, then a Mourre result holds for any $E_{0} \neq$ threshold (and $\alpha$ is twice the distance from $E_{0}$ to the threshold of next lowest energy).

To conclude (i) and (iii) in Thm. 4.1, we also need control on $[A, B]$ which requires $V_{i j}$ have more decay than in the above theorem $\left(e . g \cdot\left(1+x^{2}\right) v^{(2)}\left(h_{0}+i\right)^{-1}\right.$ $v^{(1)}\left(h_{0}+i\right)^{-1}$ and $x^{2} \nabla \nabla V^{(1)}\left(h_{0}+i\right)^{-1}$ compact wi11 do); see e.g. [51]. FroeseHerbst $[60]$ have a simple proof of Thm. 4.3. 
193

Froese-Herbst [63] have proven the following theorems using Mourne estimates:

Theorem 4.4 [63] Let $V_{i j}$ obey the hypotheses of Theorem 4.3, and suppose that $H_{\varphi}=E_{\varphi}$, with $\varphi \in L^{2}$. Let $[x]$ denote the norm of $x$ in $x$ (i.e. $\left(\Sigma m_{i} x_{i}^{2}\right)^{\frac{1}{2}}$ if $\left.\sum m_{i} x_{i}=0\right)$. Define

$$
\alpha=\sup \left\{a\left|e^{a|x|}\right|_{\varphi \in L^{2}}\right\}
$$

Then either $\alpha=\infty$ or $\alpha^{2}+E$ is a threshold.

By using results [64] which imply $\alpha=\infty$ is not allowed:

Theorem 4.5 [63] If the $V_{i j}$ obey the hypotheses of Theorem 4.3, and for all $\varepsilon>0$

$$
y \cdot \nabla V_{i j}(y) \leq \varepsilon h_{0}+C_{\varepsilon}
$$

then $H_{\varphi}=E \varphi$ has no $L^{2}$ solutions with $E>0$. 


\section{An Introduction to the Theory of Stochastic Jacobi Matrices}

In this final section, we consider another topic currently of intense interest, namely Schrödinger operators with random or almost periodic potentials. For technical simplicity, we will restrict ourselves to $\nu=1$ and we will discretize space, i.e. replace $R$ by $Z$ and $-d^{2} / d x^{2}$ by a second difference operator. See [65] for an extensive bibliography including papers dealing with the continuum case and with $\nu>1$.

We should take $h_{0}$ to be the finite difference analog of $-d^{2} / d x^{2}$, namely $\left(h_{0} u\right)(n)=\delta^{-2}[2 u(n)-u(n+1)-u(n)]$. First of a11, we take $\delta=1$ for convenience. Then, we replace $h_{0}$ by $h_{0}-2$ which won't change any spectral properties. Then we make the unitary transformation $u(n) \rightarrow(-1)^{n} u(n)$ which means that instead, we take

$$
\left(h_{0} u\right)(n)=u(n+1)+u(n-1)
$$

on $\ell^{2}(Z)$. We will study not individual operators but whole classes: Let $(\Omega, \mu)$ be a probability measure space and let $T: \Omega \rightarrow \Omega$ be an invertible, measure preserving ergodic transformation. Let $f: \Omega \rightarrow R$ be a bounded measurable function. Given $\omega \in \Omega$, define

$$
V_{\omega}(n)=f\left(T_{\omega}^{\alpha}\right)
$$

and

$$
h_{w}=h_{0}+v_{w}
$$

We ask about properties of $h_{w}$ that hold for a.e. $w$.

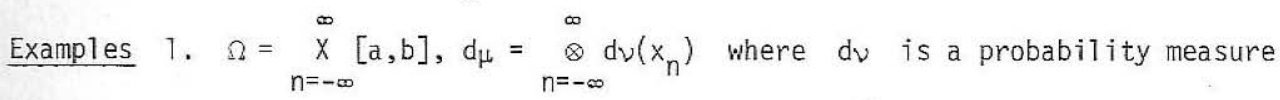
on $[a, b]$. Let $(T x)_{n}=x_{n+1}$ and $f(x)=x_{0}$. Then the variables $V_{\omega}(n)$ are precisely independent identically distributed (i.i.d.) random variables with common density $d v$. This is conventionally called "random potentials." The case $\mathrm{d} \nu(\mathrm{x})=(\mathrm{b}-\mathrm{a})^{-1} x_{(\mathrm{a}, \mathrm{b})}(\mathrm{x}) \mathrm{dx}$ is called the Anderson modet.

2. Let $\Omega$ be the $k$ torus $\left\{\left(\theta_{1}, \ldots, \theta_{k}\right) ; 0 \leq \theta<1\right\}$ with its structure as a group (addition of components, mod. 1) and Haar measure $\frac{k}{1} d \theta_{i}$. Let $f$ be 
a continuous function on $\Omega$ and let $(T \theta)_{i}=\theta_{i}+\alpha_{i}(\bmod 1)$ where $\alpha_{1}, \ldots, \alpha_{k}$ are numbers so that $1, \alpha_{7}, \ldots, \alpha_{k}$ are independent over the rationals. Then $V_{\theta}(n)=f\left(\alpha_{j} n+\theta_{j}\right)$ is quasiperiodic. A good example is $V_{\theta}(n)=\lambda \cos (2 \pi \alpha n+\theta)$ (now $\theta$ runs through $[0,2 \pi)$ ) called Hopper's equation or the almost Mathieu equation. An interesting example (see $[66,67,68]$ ) which doesn't quite fit into this framework is $V_{\theta}(n)=\lambda \tan (\pi a n+\theta)$. This is called the Moryland model and has the feature of being exactly soluble in a certain sense.

It makes sense to study the totality of the operators $\left\{h_{\psi}\right\}$ for one has the following consequence of ergodicity.

Theorem 5.1 ([69]) The following sets are constant for a.e. $\omega$ (i.e. there is a set $S \subset \Omega$ whose complement has measure zero, so that if $\omega, \omega^{\prime} \in S$ all the objects below are equal for $\omega$ and $\left.\omega^{\prime}\right): \sigma\left(h_{\omega}\right), \sigma_{a} . c .\left(h_{\omega}\right), \sigma_{p p}\left(h_{\omega}\right)(\equiv$ closure of set of eigenvalues), $\sigma_{\text {s.c. }}\left(h_{\omega}\right)$. Moreover, $\sigma_{\text {disc }}\left(h_{\omega}\right)=\phi$ and $\sigma\left(h_{\omega}\right)$ has no isolated points.

Remark $\sigma_{\text {disc }}\left(h_{\omega}\right)=\phi$ also in the higher dimensional case; it is also true in that case that $\sigma\left(h_{\omega}\right)$ has no isolated points, but this is more subtle (see $[70,71]$ ). Here are some typical results illustrating the subtle spectral properties of stochastic Jacobi matrices:

Theorem 5.2 Let $h_{\omega}$ have a random potential $\left(V_{\omega}(n)\right.$ i.i.d.'s) with $d v(x)=F(x) d x$ (supported on $[a, b])$. Then, for a.e. $w$,

$$
\operatorname{spec}\left(h_{\omega}\right)=[-2,2]+\operatorname{supp}(F)
$$

and $h_{w}$ has a complete set of eigenfunctions.

For proofs see $[69,72]$. For related continuum results, see $[73,74]$. For the study of $h_{0}+\left(1+[n !)^{-\alpha} V_{\omega}(n)\right.$, see $[75,76]$.

Theorem 5.3 Let $\left\{a_{n}\right\} \in \ell_{1}(0,1, \ldots)$ and let $h\left(a_{m}\right)=h_{0}+\sum_{m=0}^{\infty} a_{m} \cos \left(2 \pi n / 2^{m}\right)$. Then for a dense $G_{\delta}$ in $\ell_{1}, h\left(a_{m}\right)$ has a nowhere dense spectrum and for a dense set in $\ell_{1}, \sigma\left(h\left(a_{m}\right)\right)$ is both nowhere dense and purely absolutely continuous. 
See $[77,78,79]$ for proofs; see $[80]$ for a discussion of nowhere dense a.c. spectrum.

Theorem 5.4 Pick any $0<\alpha<1$. Then, there exists almost periodic potentials $V_{\omega}(n)$ so that $h_{0}+V_{\omega}(n)=h_{\omega}$ has dense point spectrum and $\sigma\left(h_{\omega}\right)$ has Hausforff dimension $\alpha$.

The basic idea is from Craig [81], al though his examples are not strictly almost periodic; those are due to Poschel [82]. See also [83].

Sarnak [84] first suggested that spectral properties should depend on Diophantine properties of $\alpha$ :

Theorem 5.5 Let $\alpha$ be an irrational number for which there exist rational approximations $p_{n} / q_{n}$ obeying $\left|\alpha-p_{n} / q_{n}\right| \leq n^{-q_{n}}$. Let $\lambda>2$. Then

$$
h_{0}+\lambda \cos (2 \pi a n+\theta)
$$

has purely singular continuous spectrum.

For a proof, see Avron-Simon [85]; important input comes from Aubry-André [86] and Gordon [87]. The set of $\alpha$ obeying the estimates is a dense $G_{\delta}$ in $R$ (of Lebesgue measure zero).

Definition A stochastic process $V_{\omega}(n)$ is called deterministic if and only if $\left\{V_{w}(n)\right\}_{n \geq 0}$ is (a.e.) a measurable function of $\left\{V_{w}(n)\right\}_{n<0}$. For example, a.p. functions yield deterministic processes; random potentials do not.

Theorem 5.6 If $h_{w}$ is a stochastic Jacobi matrix and $h_{w}$ has some a.c. spectrum (for a.e. $w$ ), then $v_{w}$ is a deterministic process.

This result in the continuum case is due to Kotani [88]; see Simon [89] for the discrete case. 


\section{References}

1. M. Reed and B. Simon, Methods of modem mathematical physics, Vot. II. Fourier analysis, self adjointness, Academic Press, 1975.

2. M. Reed and B. Simon, Methods of modem mathematical physics, Vol. III. Scattering theory, Academic Press, 1978.

3. M. Reed and B. Simon, Methods of modem mathematical physics, Vol. IV. Analysis of operators, Academic Press, 1977.

4. H. Cycon, R. Froese, W. Kirsch and B. Simon, Lectures on Schrödinger operators, in preparation.

5. M. Reed and B. Simon, Methods of modem mathematical physics, Vol. I. Functionat analysis, Academic Press, 1972.

6. T. Kato, Perturbation theory for tinear operators, Springer, 1966.

7. M. Schechter, spectra of partial differential operators, North Holland, 1971.

8. T. Kato, Schrödinger operators with singular potentials, Is. J. Math. 13 (1973), 135-148.

9. B. Simon, Maximal and minimal Schrödinger forms, J. Op. Th. I (1979), 37-47.

10. H. Leinfelder and C. Simader, Schrödinger operators with singular magnetic vector potentials, Math. Z. 176 (1981), 1-19.

11. F. Rellich, Störungstheorie der spectralzerlegung, II, Math. Ann. 116 (1939), 555-570.

12. T. Kato, Fundamental properties of Hamiltonian operators of Schrödinger type, Trans. Am. Math. Soc. 70 (1951), 195-211.

13. F. Stumel, Singulare elliptische differentialoperatoren in Hilbertschen Räumen, Math. Ann. 132 (1956), 150-176.

14. A.G. Sigalov and I.M. Sigal, Description of the spectrum of the energy operator of quantum-mechanical systems..., Theor. and Math. Phys. $\underline{5}$ (1970), 990. 
15. P. Deift, W. Hunziker, B. Simon and E. Vock, Pointwise bounds on eigenfunctions and wave packets in $\mathrm{N}$-body quantum systems, IV, Commun. Math. Phys. 64 (1978), 1-34.

16. B. Simon, Schrödinger semigroups, Bu11. AMS $\underline{7}$ (1982), 447-526.

17. M. Aizenman and B. Simon, Brownian motion and Harnack's inequality for Schrödinger operators, Comm. Pure Appl. Math. 35 (1982), 209-273.

18. Z. Zhao, Uniform boundedness of conditional gauge and Schrödinger equations, Commun. Math. Phys., to appear, and Continuity of conditioned Feynman-Kac functional and integral kernel for Schrödinger equation, Stanford preprint.

19. J. Brossard, The problem of Dirichlet for the Schrödinger operator, Institut Fourier preprint, 1984.

20. B. Simon, On the number of bound states of two-body Schrödinger operators A review, from Studies in mathematical physics, essays in honor of Valentine Bargmann, Princeton Press, 1976, pp. 305-326.

21. B. Simon, Functional integration and quantum physics, Academic Press, 1979.

22. E.H. Lieb and $W$. Thirring, Inequalities for the moments of the eigenvalues of the Schrödinger Hamiltonian and their relation to Sobolev inequalities, in Studies in mathematical physics, essays in honor of Vatentine Bargmonn, Princeton Press, 1976, pp. 269-304.

23. G. Rosenb1jum, The distribution of the discrete spectrum for singular differential operators, Dok1. Akad. Nauk SSSR 202 (1972); trans1. Soviet Math. Dok1. 13 (1972), 245-249.

24. M. Cwikel, Weak type estimates and the number of bound states of Schrödinger operators, Ann. Math. 106 (1977), 93-102.

25. E. Lieb, Bounds on the eigenvalues of the Laplace and Schrödinger operators, Bu11. AMS 82 (1976), 751-753, and Proc. 1979 AMS Honolu1u Conference.

26. B. Simon, Trace ideats and their applications, Cambridge Univ. Press, 1979. 
27. P. Li and S.T, Yau, On the Schrödinger equation and the eigenvalue problem, Commun. Math. Phys. 88 (1983), 309.

28. C. Fefferman, The uncertainty principle, Bul1. Am. Math. Soc. 9 (1983), 129.

29. M. Birman and V.V. Borzov, On the asymptotics of the discrete spectrum of some singular differential operators, Topics in Math. Phys. $\underline{5}$ (1972), 19-30.

30. A. Martin, Bound states in the strong coupling limit, Helv. Phys. Acta. $\underline{45}$ (1972), 140-148.

31. H. Tamura, The asymptotic eigenvalue distribution for nonsmooth elliptic operators, Proc. Japan Acad. 50 (1974), 19-22.

32. M. Klaus and B. Simon, Coupling constant threshold in non-relativistic quantum mechanics, II. Two body thresholds in $\mathrm{N}$-body systems, Commun. Math. Phys. 78 (1980), 153-168.

33. A. Persson, Bounds for the discrete part of the spectrum of a semibounded Schrödinger operator, Math. Semd. $\underline{8}$ (1960), 143-153.

34. S. Agmon, Lectures on exponential decay of solutions of second onder elliptic equations. Bounds on eigenfunctions of $N$-bods Schrodinger operators, Mathematical Notes, Princeton Univ, Press, Princeton, NJ 1982.

35. S. Agmon, On exponential decay of solutions of second order elliptic equations in unbounded domains, Proc. A. Pleijel Conf.

36. L. Garding, On the essential spectrum of Schrödinger operators, J. Func. Anal. $\underline{52}$ (1983), 1-10.

37. W. Hunziker, On the spectra of Schrödinger multiparticle Hamiltonians, Helv. Phys. Acta $\underline{39}$ (1966), 451-462.

38. C. van Winter, Theory of finite systems of particles, I. Mat. Fys. Skr. Danske Vid. Selsk I (1964), 1-60.

39. G. Zhislin, Discussion of the spectrum of the Schrödinger operator for systems of many particles, Trudy. Mosk. Mat. Obs. $\underline{9}$ (1960), 81-128. 
40. V. Enss, A note on Hunziker's theorem, Commun. Math. Phys. 52 (1977), 233.

41. B. Simon, Geometric methods 111 multiparticle quantum systems, Commun. Math. Phys. 55 (1977), 259-274.

42. I.M. Sigal, Geometric methods in the quantum many-body problem. Nonexistence of very negative ions, Commun. Math. Phys. 85 (1982), 309-324.

43. G.M. Zhislin, On the finiteness of the discrete spectrum of the energy operator of negative atomic and molecular ions, Theor. Math. Phys. $\underline{7}$ (1971), 571-578.

44. M.A. Antonets, G.M. Zhislin and J.A. Sheresherskii, On the discrete spectrum of the Hamiltonian of an n-particle quantum system, Theor. Math. Phys. 16 (1973), 800.

45. M.B. Ruskai, Absence of discrete spectrum in highly negative ions, I, II. Commun. Math. Phys. 82 (1982), 457; 85 (1982), 325.

46. E.H. Lieb, Bound on the maximum negative ionization of atoms and molecules, Phys. Rev. A, to appear.

47. E.H. Lieb, I.M. Sigal, B. Simon and W. Thirring, Asymptotic bulk neutrality of large $Z$ ions, Phys. Rev. Lett., to appear.

48. P. Deift and B. Simon, A time-dependent approach to the completeness of multiparticle quantum systems, Commun. Pure App1. Math. 30 (1977), 573-583.

49. E.B. Davies, On Enss' approach to scattering theory, Duke Math. J. 47 (1980), $171-185$.

50. P. Perry, Scattering theory by the Enss method, Mathematical Reports, Vol. I, 1983.

51. P. Perry, I.M. Sigal and B. Simon, Spectral analysis of multiparticle Schrödinger operators, Ann. Math. 114 (1981), 519-567.

52. L. Faddeev, Mathematical aspects of the three body problem in quantum scattering theory, Steklov Institute, 1963.

53. V. Enss, Topics in scattering theory for multiparticle quantum mechanics, to 
appear in Boulder IAMP Proceedings.

54. E. Mourre, Operateurs conjugués et propriêtés de propagation, II, preprint.

55. G. Hagedorn, Asymptotic completeness for classes of two, three, and four particle Schrödinger operators, Trans. AMS 258 (1980), 1-75.

56. I.M. Sigal, On quantum mechanics of many-body systems with dilation-analytic potentials, Bul1. AMS $\underline{84}$ (1978), 152-154.

57. E. Mourre and I.M. Sigal, Phase space analysis and scattering theory for $\mathrm{N}-$ particle systems, preprint.

58. I.M. Sigal, Mathematical foundations of quantum scattering theory for multiparticle systems, Mem. Am. Math. Soc. 209 (1978).

59. E. Mourre, Absence of singular spectrum for certain self-adjoint operators, Commun. Math. Phys. 78 (1981), 391.

60. R. Froese and I. Herbst, A new proof of the Mourre estimate, Duke Math. J. $\underline{49}$ (1982), 1075 .

61. E. Mourre, Operateurs conjugués et propriétés de propagation, I, Commun. Math. Phys. 91 (1983), 279.

62. A. Jensen, E. Mourre and P. Perry, Multiple commutator estimates and resolvent smoothness in quantum scattering theory, preprint, 1983.

63. R. Froese and I. Herbst, Exponential bounds and absence of positive eigenvalues for N-body Schrödinger operators, Commun. Math. Phys. 87 (1982), 429.

64. R. Froese, I. Herbst, M. Hoffmann-Ostenhof and T. Hoffmann-Ostenhof, $L^{2}$-exponential lower bounds to solutions of the Schrodinger equation, Commun. Math. Phys. 87 (1982), 265-286.

65. B. Simon and B. Souillard, Franco-American meeting on the mathematics of random and almost periodic potentials, J. Stat. Phys., to appear.

66. R. Prange, D. Grempel and S. Fishman, A solvable model of quantum motion in an incommensurate potential, Phys. Rev., in press; Localization in an incommensurate potential: An exactly solvable model, Phys. Rev. Lett. 49 (1982), 833. 
67. B. Simon, Almost periodic Schrödinger operators, IV. The Maryland model, Ann. Phys., to appear.

68. L. Pastur and A. Figotin, Localization in an incommensurate potential: Exactly solvable multidimensional model, JETP Lett. 37 (1983), 686; paper to appear in Commun. Math. Phys.

69. H. Kunz and B. Souillard, Sur le spectre des opérateurs aux différences finies aléatoires, Commun. Math. Phys. 78 (1980), 201-246.

70. W. Craig and B. Simon, Log HöTder continuity of the integrated density of states for stochastic Jacobi matrices, Commun. Math. Phys. 90 (1983), 207-218.

71. F. Delyon and B. Souillard, Remark on the continuity of the density of states of ergodic finite difference operators, Commun. Math. Phys., to appear.

72. F. Delyon, H. Kunz and B. Souillard, One dimensional wave equations in disordered media, J. Phys. Al6 (1983), 25.

73. I. Goldsheid, S. Molchanov and L. Pastur, A pure point spectrum of the stochastic and one dimensional Schrödinger equation, Funct. Ana1. Appl. 11 (1977), 1-10.

74. R. Carmona, Exponential localization in one dimensional disordered systems, Duke Math. J. 49 (1982), 191.

75. B. Simon, Some Jacobi matrices with decaying potential and dense point spectrum, Commun. Math. Phys. 87 (1982), 253-258.

76. F. Delyon, B. Simon and B. Souillard, From power law localized to extended states in a disordered system, preprint.

77. J. Moser, An example of a Schrödinger equation with an almost periodic potential and nowhere dense spectrum, Comm. Math. Helv. 56 (1981), 198.

78. V. Chulaevsky, On perturbations of a Schrödinger operator with periodic potentia1, Russian Math. Surveys 36, №. 5 (1981), 143.

79. J. Avron and B. Simon, Almost periodic Schrödinger operators, I. Limit periodic potentials, Commun. Math. Phys. 82 (1982), 101-120. 
80. J. Avron and B. Simon, Transient and recurrent spectrum, J. Func. Ana 1. 43 (1981), 1-31.

81. W. Craig, Pure point spectrum for discrete almost periodic Schrödinger operators, Commun. Math. Phys. 88 (1983), 113-131.

82. J. Pöschel, Examples of discrete Schrödinger operators with pure point spectrum, Commun. Math. Phys. 88 (1983), 447-463.

83. J. Bellissard, R. Lima and E. Scoppola, Localization in v-dimensional incommensurate structures, Commun. Math. Phys. 88 (1983), 465-477.

84. P. Sarnak, Spectral behavior of quasi periodic potentials, Commun. Math. Phys. 84 (1982), 377-401.

85. J. Avron and B. Simon, Almost periodic Schrödinger operators, II. The integrated density of states, Duke Math. J. 50 (1983), 369-391.

86. S. Aubry and G. Andre, Analyticity breaking and Anderson 1ocalization in incommensurate lattices, Ann. Israel Phys. Soc. $\underline{3}$ (1980), 133.

87. A. Gordon, Usp. Math. Nauk. 31 (1976), 257.

88. S. Kotani, Proceedings of the Conference on Stochastic Processes, Kyoto, 1982.

89. B. Simon, Kotani theory for one dimensional stochastic Jacobi matrices, Commun. Math. Phys. 89 (1983), 227. 